\title{
Glycine-Chitosan-Based Flexible Biodegradable Piezoelectric Pressure Sensor
}

\author{
Ensieh S. Hosseini, Libu Manjakkal, Dhayalan Shakthivel, and Ravinder Dahiya* \\ Cite This: ACS Appl. Mater. Interfaces 2020, 12, 9008-9016 \\ Read Online
}

ABSTRACT: This paper presents flexible pressure sensors based on free-standing and biodegradable glycine-chitosan piezoelectric films. Fabricated by the self-assembly of biological molecules of glycine within a water-based chitosan solution, the piezoelectric films consist of a stable spherulite structure of $\beta$-glycine (size varying from a few millimeters to 1 $\mathrm{cm}$ ) embedded in an amorphous chitosan polymer. The polymorphic phase of glycine crystals in chitosan, evaluated by X-ray diffraction, confirms formation of a pure ferroelectric phase of glycine ( $\beta$-phase). Our results show that a simple solvent-casting method can be used to prepare a biodegradable $\beta$-glycine/chitosan-based piezoelectric film with sensitivity $\left(\sim 2.82 \pm 0.2 \mathrm{mV} \mathrm{kPa}^{-1}\right)$ comparable to those of nondegradable commercial piezoelectric materials. The measured capacitance of the $\beta$-glycine/chitosan film is in the range from 0.26 to $0.12 \mathrm{nF}$ at a frequency range from $100 \mathrm{~Hz}$ to $1 \mathrm{MHz}$, and its dielectric constant and loss factor are 7.7 and 0.18 , respectively, in the high impedance range under ambient conditions. The results suggest that the glycine-chitosan composite is a promising new biobased piezoelectric material for biodegradable sensors for applications in wearable biomedical diagnostics.

KEYWORDS: $\beta$-glycine, chitosan, piezoelectric, biodegradable pressure sensor, wearable electronics

\section{INTRODUCTION}

With the advent of wearable systems for health monitoring, there is a tremendous need for the development of biodegradable self-powered devices to monitor the physiological state. ${ }^{1,2}$ To this end, piezoelectric materials are appealing as they can generate electrical charges under mechanical stress and hence can be used for force/pressuresensing applications. ${ }^{3}$ The commonly used piezoelectric materials, such as lead zirconate titanate (PZT), lithium niobate, etc., exhibit strong piezoelectric properties, but they also contain toxic, nonrenewable, and nonbiodegradable components. In addition, synthesizing such materials often requires high temperature and an electrical poling process. ${ }^{4}$ Such steps make it difficult to fabricate biodegradable sensors on flexible substrates for wearable health-monitoring applications, such as wound healing, where there is a need to measure the sub-bandage pressure to monitor the wound healing rate., In such cases, pressure sensors integrated with a compression bandage can be helpful, and their biodegradability is desired for hygiene purposes. Exploring this aspect, the need for new biodegradable materials is increasingly becoming important for wearable systems.

Biobased piezoelectric materials, such as amino acids, collagen, cellulose, and peptide nanotubes, have raised significant interest in recent years due to their biocompatibility, renewable nature, low cost, and simple and low-temperature processing. $^{7-9}$ Piezoelectric force sensors and power generators based on biomaterials such as cellulose nanofibril ${ }^{10}$ and viruses ${ }^{11}$ have been reported recently. The random distribution of cellulose nanofibrils in the film and low stability of selfassembled viruses on the substrate in these works limit their use in real applications. Further, most of the biomaterials have weak macroscale piezoelectric responses, and it is difficult to obtain a uniform and scalable unidirectional polarized structure. This is notwithstanding other recent works where by using a high electric field or by controlling the peptide solution and pulling speeds of the substrate, the aligned (both vertically and horizontally) piezoelectric peptide nanotubes have been shown to have significantly improved output voltage. ${ }^{7,12}$ In this regard, the amino acids (e.g., glycine) are attractive as they can be self-assembled in organized polar crystalline structure at large scale. They can be grown as unidirectional crystals, and this makes them suitable for fabrication of pressure sensors. Processing in aqueous media, low cost, and high throughput are a few other advantages of these materials.

Glycine is the simplest amino acid which has been studied extensively in drug administration. ${ }^{13}$ Glycine can be crystallized in three polymorphic phases $(\alpha, \beta$, and $\gamma)$ under ambient conditions. $\gamma$-Glycine and $\beta$-glycine are known to have

Received: November 23, 2019

Accepted: February 3, 2020

Published: February 3, 2020 


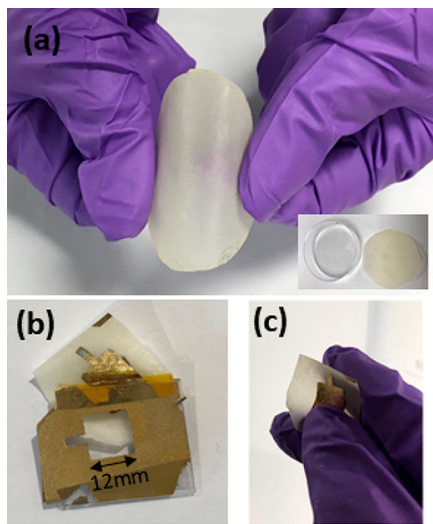

(d)

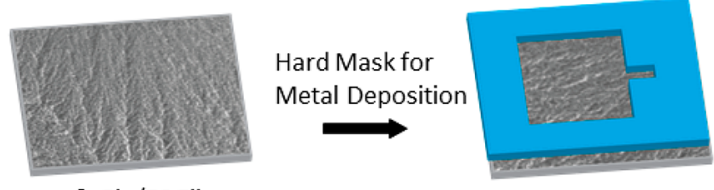

$\beta-$ Gly/CS Film

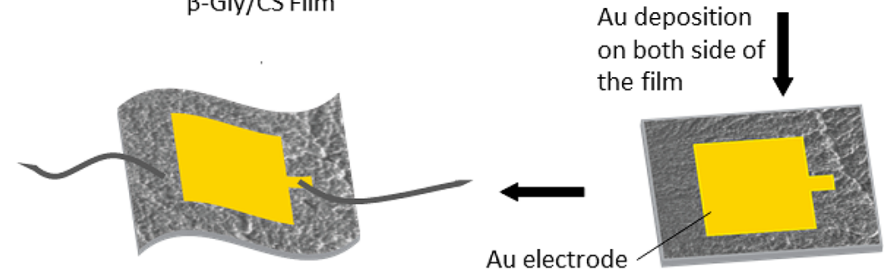

Figure 1. Optical images of the (a) glycine/chitosan (Gly/CS) film and (b, c) deposited Au electrodes on film and fabricated flexible sensor. (d) Schematic representation of the fabrication process of the flexible piezoelectric Gly/CS-based pressure sensor.

piezoelectric properties due to a noncentrosymmetric polar structure, and the $\alpha$-phase has a centrosymmetric structure without piezoelectricity. ${ }^{14,15} \mathrm{~A}$ few recent reports have presented experimental evidence of the piezoelectric and ferroelectric properties of glycine $e^{9,16}$ with a very high piezoelectric coefficient $\left(d_{16}=174 \mathrm{pm} \mathrm{V}^{-1}\right)$ observed in the metastable $\beta$-phase. ${ }^{17}$ However, due to the thermodynamic instability of the $\beta$-phase, not much has been reported on the application of the piezoelectric properties of the $\beta$-phase. Most of the research thus far has focused on stabilization of the polymorphs with techniques such as crystal growth in nanoscale crystallization chambers, ${ }^{18,19}$ on patterned substrate $^{20}$ or on Pt-coated Si substrates. ${ }^{21}$ In all of these reported methods, the $\beta$-crystals were micro/nanoscale and/or are usually in a mixture with the other phases of glycine. In another recent work, piezoelectricity/ferroelectricity on selfassembled microislands of $\beta$-glycine on the $\mathrm{Si}$ substrate has been reported. ${ }^{22}$ Although the reported microcrystals were quite stable and grown on the same crystallographic direction, it is difficult to use them for any device fabrication due to their small size and stability while in contact with the rigid silicon substrate. To overcome these challenges, a flexible piezoelectric composite of glycine crystals and elastomeric dielectric polymers is required.

In this work, we report the uniform and highly stable $\beta$ glycine films fabricated by growing glycine crystals in a chitosan matrix to obtain a flexible piezoelectric composite. To the best of our knowledge, the flexible piezoelectric sensors of glycine amino acids have not been reported so far. The bioresorbable chitosan polymers display a series of unique properties due to which they have been widely used in biomedical applications, such as temporary interventions inside the human body. ${ }^{23,24}$ Due to its biodegradable, biocompatible, nontoxic nature and excellent antibacterial properties, chitosan has also been used in wound dressing. ${ }^{25-28}$ Chitosan could also increase the organization of collagen fibers in a collagenchitosan film and enhance the piezoelectric response of the film. ${ }^{29}$ Crystallization of some synthetic polymer in chitosan and the overall kinetics of crystallization have been reported in past, $^{30}$ and it is known that depending on the nature of the polymer matrix and interaction between the chitosan and primary nuclei, the crystals grow into various morphologies and structures. ${ }^{31}$ However, there is no report on the selfassembly of biodegradable organic crystals within chitosan polymer. In this work, glycine powder was dissolved in chitosan polymer solution and films of $\beta$-glyine/chitosan $(\beta$ -
Gly/CS) were prepared via a simple solution-casting technique followed by drying at room temperature. By controlling the composition of glycine and chitosan, we successfully synthesized the crystallographically oriented and stable $\beta$ glycine crystals within chitosan. Here, chitosan is used as the matrix material, and the grown bio-organic glycine crystals are embedded in the chitosan film. The morphology and microstructure of the film were studied using optical imaging and scanning electron microscopy (SEM). The dielectric properties of the material including the impedance, dielectric constant, loss factor, and capacitance of the fabricated film were measured by impedance spectroscopic analysis. Finally, the flexible biodegradable piezoelectric films were used as functional materials in the piezoelectric sensors, and their sensitivity was measured under dynamic pressure $(5-60 \mathrm{kPa})$.

\section{EXPERIMENTAL SECTION}

2.1. Fabrication of Glycine/Chitosan Composites Film. Low molecular weight chitosan and glycine powder were obtained from Sigma Aldrich. A 1.5 wt \% chitosan solution was prepared by mixing chitosan powder with a $1 \% \mathrm{v} / \mathrm{v}$ acetic acid aqueous solution. A series of glycine/chitosan composite solutions, with glycine to chitosan ratios $(\mathrm{w} / \mathrm{w})$ of $0.4: 1,0.8: 1,1.2: 1$, and $2.7: 1$, was prepared by dissolving different amounts of glycine powder in the prepared $1.5 \mathrm{wt}$ $\%$ chitosan solution by stirring. The well-mixed glycine/chitosan solution was drop casted onto the different substrates, such as $\mathrm{Si}$ wafer, glass, and inside a polystyrene Petri dish, followed by drying at room temperature for 24-48 h. After complete drying, the morphology and crystalline phase of the film that was obtained on different substrates were investigated. The effect of different concentrations of glycine on the final morphology and crystallinity of film was investigated and compared with plain chitosan. In addition, glycine crystals were grown from a $1 \% \mathrm{v} / \mathrm{v}$ acetic acid aqueous solution on the same concentration range and without chitosan to investigate the effect of the chitosan on the polymorph selectivity and controlled crystallinity of glycine molecules.

2.2. Fabrication of Glycine/Chitosan Sensors. For measurement of the impedance properties and piezoelectric sensitivity of the film under the applied pressure, the film is required to be sandwiched between two electrodes. A $5 \mathrm{~g}$ amount of the solution with a glycine to chitosan ratio of $0.8: 1$ was drop casted on a $60 \mathrm{~mm}$ polystyrene Petri dish. The solution was dried at room temperature by evaporation, and glycine molecules formed an ordered crystalline structure inside the chitosan film. The grown glycine/chitosan film could be easily peeled off from the Petri dish (Figure 1a). Subsequently, a gold electrode $(\mathrm{Ti} / \mathrm{Au})$ with a thickness of $10 / 90$ $\mathrm{nm}$ was deposited on both sides of the glycine/chitosan film using a hard mask and electron-beam evaporation technique, as shown in Figure $1 \mathrm{~b}$ and $1 \mathrm{c}$. Then wires were connected to the top and bottom 

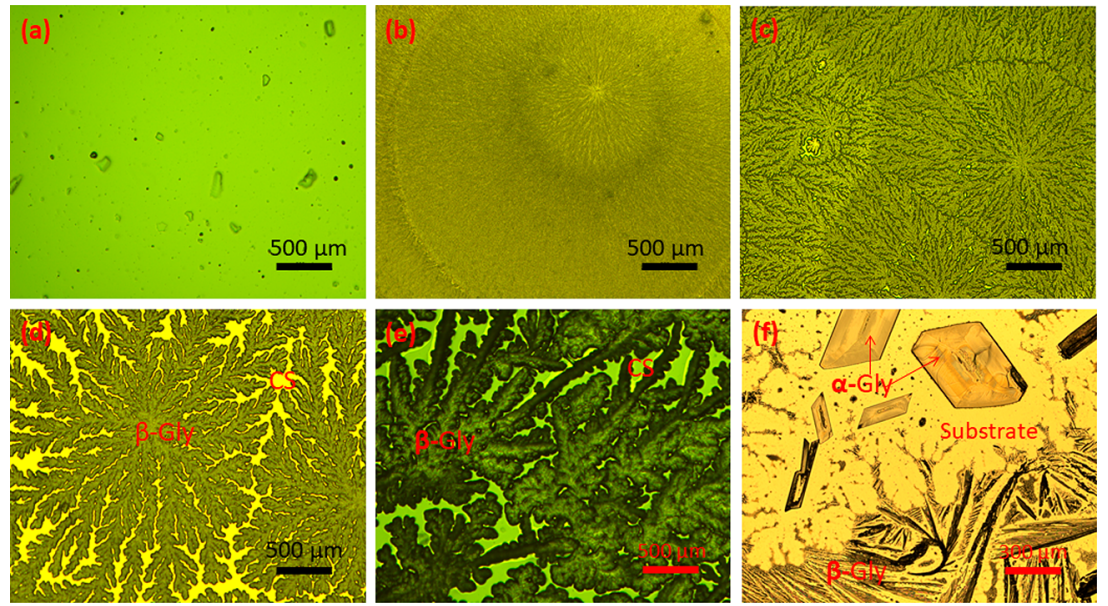

Figure 2. Optical microscopy images of (a) plain chitosan and spherulite morphology of glycine in $\beta$-Gly/CS film, grown from blends of various glycine to chitosan ratios: (b) 0.4:1, (c) 0.8:1, (d) 1.2:1, and (e) 2.7:1. (f) Optical micrograph of $\alpha$ and $\beta$ polymorphs observed from evaporated water-based glycine solution.

electrodes, and the device was encapsulated with Kapton tape. Figure $1 \mathrm{~d}$ shows the fabrication process of the glycine/chitosan piezoelectric sensor. The effective device area where the glycine/chitosan film was in contact with both electrodes was $144 \mathrm{~mm}^{2}$, and the film thickness was $38 \mu \mathrm{m}$. The fabricated sensor shows good flexibility (Figure 1c) and robustness, thus confirming its suitability in wearable systems.

2.3. Glycine/Chitosan Film and Sensor Characterization. The surface morphology of the glycine/chitosan film was evaluated using an optical microscope (Nikon, Eclipse LV100ND). The microstructure of glycine inside chitosan films and the cross section of the glycine/chitosan composite film were evaluated with scanning electron microscopy (FEI Nova NanoSEM). The crystalline phase of glycine inside chitosan was determined by X-ray diffraction (XRD) (PANalytical X'Pert PRO MPD diffractometer) using $\mathrm{Cu} \mathrm{K} \alpha$ radiation $(\lambda=1.54059 \AA)$ over the range of $10-50^{\circ}$ with steps of $0.02^{\circ}$. The diffractometer uses JCPDS reference files, numbers 00002-0687 $(\alpha)$, 00-002-0171 $(\beta)$, and 00-006-0230 $(\gamma)$, for glycine phases. The thickness of the fabricated glycine/chitosan film was measured using a profilometer (Bruker DektakXT stylus profiler). The dielectric properties, capacitance, and impedance of the glycine/ chitosan film were evaluated by impedance spectroscopic analysis using an E4980AL LCR meter.

The output voltages generated by the piezoelectric glycine in chitosan, under soft manual touch, were measured with an Agilent $34461 \mathrm{~A}$ voltmeter controlled with the LabVIEW program. A TIRA vibration system (S50018) that periodically generates compressive loads was used for measuring the sensor's sensitivity. The sensors were compressed by the mechanical shaker that delivered a sinusoidal force input, and the output voltage generated under the applied pressure was measured using an oscilloscope (Keysight, MSO$\mathrm{X} 4154 \mathrm{~A})$. The sensitivity is defined here as the voltage generated by the sensor divided by the pressure applied to excite the sensor $(\mathrm{mV}$ $\mathrm{kPa}^{-1}$ units).

\section{RESULTS AND DISCUSSION}

3.1. Glycine/Chitosan Film Structural Characterization. $\beta$-Glycine was crystallized by casting and the evaporation technique from the aqueous chitosan solution with different percentages of glycine as shown in Figure 1a. The crystallization duration for all samples (0.4:1, 0.8:1, 1.2:1, and 2.7:1 glycine:chitosan ratios) varied from 24 to $48 \mathrm{~h}$ depending on the initial concentration of glycine, and the composites with a lower concentration of glycine dried faster. We observed that the substrate did not have any effect on the film properties, and the film grown on different substrates with the same concentration of glycine had a similar morphology. It is known that polymeric and nonpolymeric materials often crystallize as spherulites when crystallized from viscous fluids. ${ }^{32}$ Different morphologies of spherulites appeared in the films casted from the viscous solutions with 0.4:1, 0.8:1, 1.2:1, and 2.7:1 ratios of glycine to chitosan as presented in Figure 2. Figure 2a shows the optical image of plain chitosan, and Figure $2 b, 2 c$, and $2 \mathrm{~d}$ reveals three typical morphologies of spherulites in the film casted from glycine:chitosan with ratios of 0.4:1, $0.8: 1$, and 1.2:1 respectively. For a higher ratio of glycine:chitosan (2.7:1), thick fibrillar crystalline regions of glycine are observed in amorphous regions of chitosan, and they are randomly oriented in the film (Figure. 2e). Morphological changes of the spherulite of glycine embedded in chitosan polymer with an increasing amount of glycine in the blends show different rates of crystallization. At a lower ratio of glycine to chitosan $(0.4: 1,0.8: 1)$, the film dried faster with an increasing number of nuclei. Following the nucleation, growth occurring under controlled diffusion of glycine molecules through the solution, the higher number of spherulite nuclei leads to a faster growth rate. A faster crystallization growth rate will cause formation of smaller and more branching fibrils as seen in Figure $2 b$ and $2 c$. With increased glycine ratios in the film-forming solution (1.2:1 and 2.7:1), the number of nuclei decrease and the growth rate reduces. At these higher ratios the width of the fibrils is larger and did not fill up the space, leading to many voids. Therefore, the fibrils in the lower glycine ratio piled up much more tightly than those in the higher. ${ }^{33}$ Due to the larger rate of nucleation and growth at a lower concentration of glycine, the size of the spherulite decreases whereas their number per unit volume increases (Figure $2 b$ ), while at a higher concentration, the number of glycine nuclei decreases and the size of each spherulite increases (Figure 2d). Glycine crystals was grown with the same concentration range in $1 \% \mathrm{v} / \mathrm{v}$ acetic acid aqueous solution without chitosan to confirm the effect of chitosan on polymorph selectivity of glycine molecules. They have been observed in two morphologies, mainly large bipyramid crystals and part of them in a fibril shape as shown in Figure $2 \mathrm{f}$.

The polymorphic phase of the glycine crystals embedded in chitosan was characterized by XRD, and it is compared with plain chitosan XRD. The XRD patterns of glycine/chitosan 

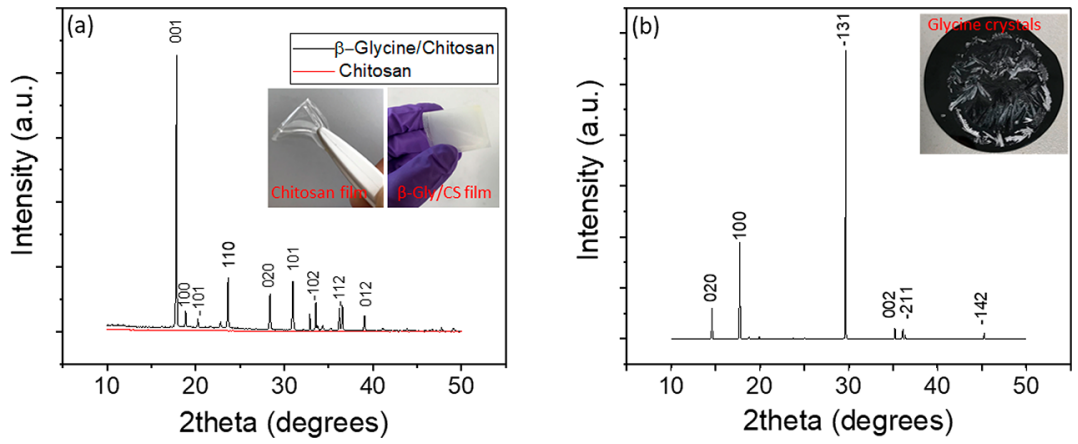

Figure 3. (a) XRD pattern of pure chitosan film (red) and glycine/chitosan film (black) with a 0.8:1 ratio. (Inset) Optical image of chitosan (left) and glycine-chitosan film (right). (b) XRD pattern of glycine crystal grown in a water-based acidic solution without chitosan. (Inset) Optical image of the glycine crystals grown without chitosan).

confirmed the formation of crystals of piezoelectric $\beta$-phase structure inside chitosan (Figure 3a, black curve). The positions of the peaks in the XRD spectrum were found to be in a good agreement with the data available in the JCPDS database for $\beta$-glycine. Various planes of reflections were indexed in XRD, as shown on Figure. 3a. The XRD spectrum for plain chitosan (in red in Figure 3a) exhibits a very broad peak and does not show any sharp peak due to the amorphous structure of chitosan. The high-intensity sharp XRD peak of the glycine/chitosan sample shows perfect crystallization of glycine. This result revealed that chitosan effectively modulated the kinetics of the self-assembly of glycine molecules in the crystalline structure. XRD of the same sample after a few months showed no changes and phase transformation. The stability of $\beta$-glycine may come from embedding crystals inside the chitosan polymer. Also, there is a possible electrostatic interaction and hydrogen-bond formation between molecular polar groups (amine and hydroxyl group) of glycine with the functional group of chitosan. ${ }^{34}$ Films grown on the different substrates with the same composition showed a similar morphology and crystallinity, which means the structure of the bottom substrate does not have any effect on the film properties. The XRD results show formation of a thermodynamically more stable phase of glycine ( $\alpha$ phase) in the absence of chitosan (Figure. $3 b$ ). Optical images of the glycine crystals in the absence of chitosan are shown in Figure $2 \mathrm{f}$ and in the inset of Figure $3 b$.

Even though the XRD result confirmed formation of the piezoelectric $\beta$-phase of glycine in all glycine/chitosan films (i.e., from 0.4:1 to 2.7:1 ratios, SI Figure S1), due to the high crystallinity of glycine the samples with a higher glycine content were less flexible. For all other measurements, we focused on the film with a $0.8: 1$ glycine:chitosan ratio since at this concentration we obtained flexible films with significant piezoelectricity. Further, the film at this concentration is uniform and highly flexible and applying the electrode is easier. Thus, for sensor characterization of the film, we considered $0.8: 1$ as the optimum ratio of glycine relative to chitosan.

It is known that crystallization occurs in supersaturated solutions. In glycine/chitosan blends, the supersaturated phase and crystallization from these solutions will occur during evaporation of solvent. $\beta$-Glycine is the thermodynamically less stable phase of glycine; therefore, fast crystallization should lead to formation of $\beta$-phase. ${ }^{19}$ Since the concentration of glycine in the chitosan solution is lower than the saturation level, the glycine/chitosan blend solution reaches a supersaturated condition just before complete drying of the film.
Therefore, the crystallization process is very quick, and mainly the thermodynamically less stable $\beta$-phase of glycine has been observed. In addition, the crystal nuclei and polymer interface affect the nucleation rate, and the viscous matrix formed by chitosan around the crystals stabilized the formed $\beta$-glycine and does not allow phase transformation by time.

SEM images of the surface of a $0.8: 1$ ratio $\beta$-glycine/ chitosan $(\beta$-Gly/Cs) film, shown in Figure $4 a-c$, clearly indicate formation of a spherulite morphology structure. The impinging spherulites with a linear interface between them indicate that these spherulites nucleated simultaneously (Figure $4 \mathrm{a}$ and $4 \mathrm{~b}$ ). Each of these spherulite are like a single crystal with a nucleus in the center, and the fibrils of the spherulite radiate out dendritically from a central nucleus, as

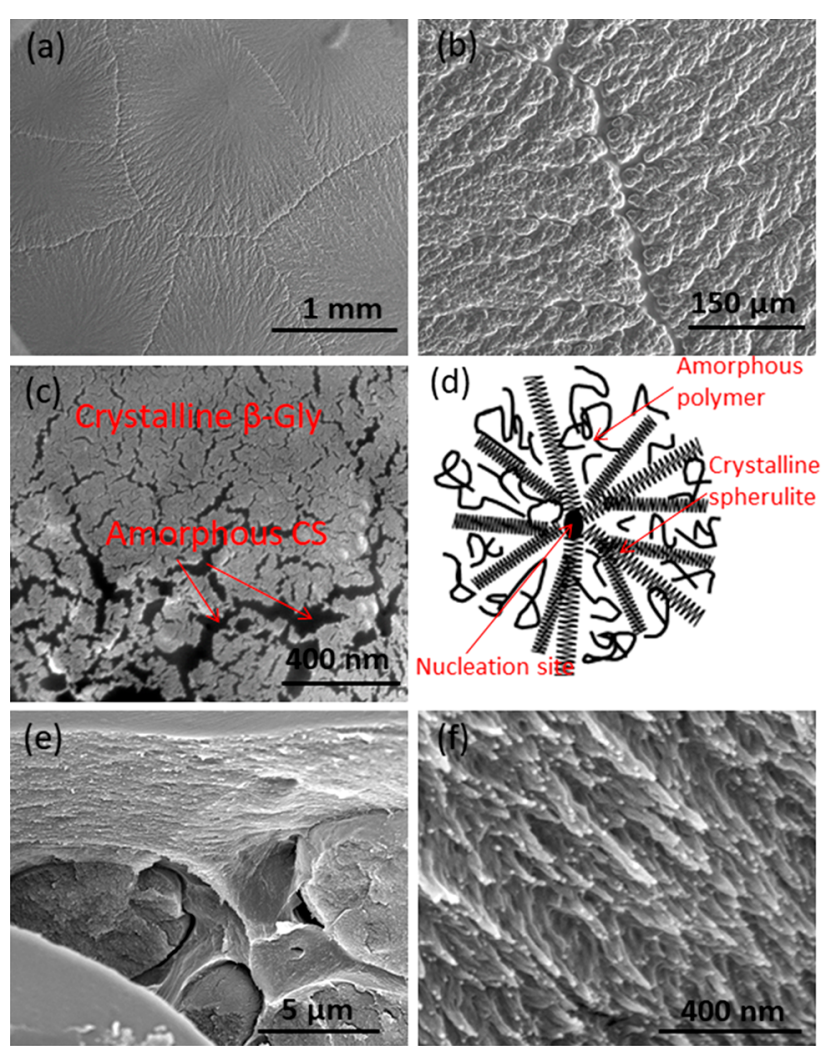

Figure 4. $(\mathrm{a}-\mathrm{c})$ SEM images of spherulites of $\beta$-glycine crystallized inside chitosan at ambient conditions with a $0.8: 1$ glycine/chitosan ratio. (d) Schematic view of spherulite crystallization. (e, f) Crosssectional SEM image of the $\beta$-Gly/CS film. 

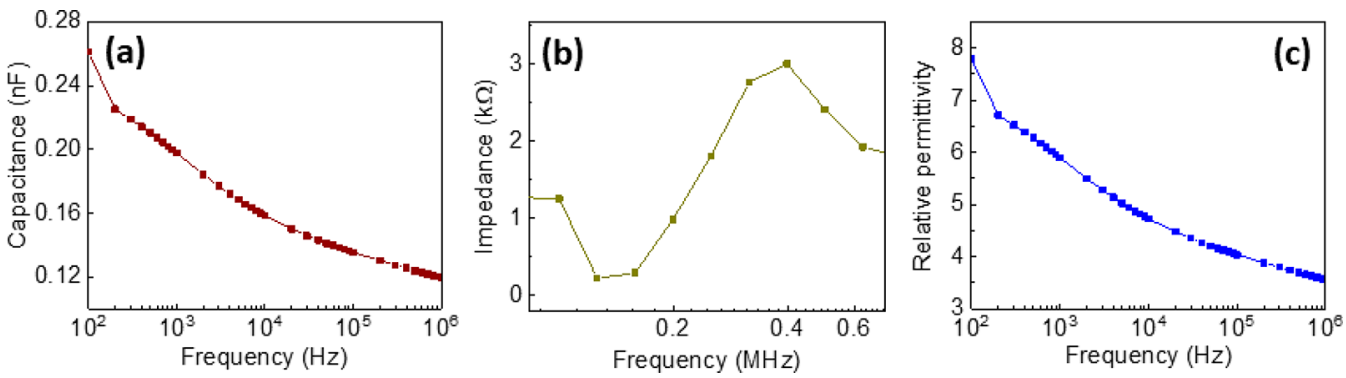

Figure 5. Impedance spectroscopic analysis of a $\beta$-Gly/CS film for a potential of $10 \mathrm{mV}$ : (a) Capacitance versus frequency; (b) variation of impedance with frequency and the piezoelectrically coupled resonance and antiresonance peaks observed at $125.89 \mathrm{~Hz}$ and $398.11 \mathrm{kHz}$, respectively; (c) variation of relative permittivity versus frequency.

shown schematically in Figure 4d. ${ }^{35}$ This occurs by creation of the spherulite core by primary nucleation, followed by the radial growth of fibrillar crystals at a constant rate. The radius of these spherulites increases linearly with time until growth is stopped by spherulite impingement. Primary nucleation is heterogeneous and being controlled by the polymer surface that provide a surface upon which nucleation can occur faster. Radial growth is accompanied by branching or cloning of crystallites to fill the space. The reason for the faster rate is a lower surface energy provided by the chitosan, resulting in a reduced size for a critical nucleus. The boundary between them can be called domain boundaries. The spherulites contain crystal lamellae which are connected to each other by amorphous regions of chitosan (Figure $4 c$ and $4 d$ ). There were always a very few spherulites, and the size of these spherulites can reach a few millimeters as shown in Figure 4a, so that they can be seen even by the naked eye.

A cross-sectional SEM image of $\beta$-Gly/CS (Figure $4 \mathrm{e}$ and 4f) reveals the homogeneous growth of $\beta$-glycine with a needle-like spherulite structure. The growth of $\beta$-glycine occurred in the bulk chitosan where it is held compactly in the amorphous matrix. Since the crystals grow in a layer of chitosan, the structure of the bottom substrate does not have any effect on crystallization as mentioned above and the film is named as outstanding. In fact, chitosan affects the polymer selectivity and crystallization similar to the case where chitosan is used as the substrate for crystallization. ${ }^{36}$

3.2. $\beta$-Gly/CS Film Dielectric Properties. The $\beta$-Gly/CS film (0.8:1 ratio) is sandwiched between two $\mathrm{Au}$ films as shown in Figure $1 \mathrm{~b}$ and 1c. The dielectric properties of the prepared sample were evaluated, and the results are shown in Figure 5. The capacitance of the $\beta$-Gly/CS film sample varies from 0.26 to $0.12 \mathrm{nF}$ with increasing frequency from $100 \mathrm{~Hz}$ to $1 \mathrm{MHz}$, as shown in Figure 5a. The piezoelectric nature of the film is further confirmed from the impedance plot at a highfrequency range in Figure $5 \mathrm{~b}$. The piezoelectrically coupled resonance is observed at a frequency of $125.89 \mathrm{kHz}$, and antiresonance is at $398.11 \mathrm{kHz}$ (shown in Figure $5 \mathrm{~b}$ ). The observed values of resonance and antiresonance in the $\beta$-Gly/ CS film are comparable to those observed for $\beta$-glycine pure crystals (resonance is at $409 \mathrm{kHz}$, and antiresonance is at 1626 $\mathrm{kHz}^{17}$ ). From the impedance plot, it was found that the ac conductivity of the glycine is low at the low-frequency range (shown in Figure S2), which is due to its crystallographic structure where the variable range of the ion-hopping mechanism occurred. The glycine molecules are generally in the form zwitterions $\mathrm{NH}_{3}{ }^{+} \mathrm{CH}_{2} \mathrm{COO}^{-}$with a hydrogen bond between them in the crystalline structure. Here, the proton ion hopping starts from the $\mathrm{NH}_{3}^{+}$to the $\mathrm{COO}^{-}$group and then from $\mathrm{COOH}$ to $\mathrm{NH}_{2} \cdot{ }^{37}$ The observed higher ionic conductance of the material at high frequency could be due to this proton-hopping mechanism. ${ }^{37}$ Further, the observed increase in conductivity with frequency is due to the grain or bulk conductivity of the material. These observations indicate that the conductance is due to mixed ionic and electronic conduction. Further, we obtained the dielectric constant (relative permittivity, $\varepsilon_{r}$ ) of the material using the expression

$$
\varepsilon_{r}=\frac{\varepsilon_{1}}{\varepsilon_{0}}=\frac{C d}{\varepsilon_{0} A}
$$

where $\varepsilon_{1}$ is the permittivity of the sample, $\varepsilon_{0}$ is the permittivity of the free space, which is equal to $8.85 \times 10^{-12} \mathrm{~F} / \mathrm{m}$, and $\varepsilon_{1}$ is measured from the capacitance and the dimension of the sample. The fabricated samples have a thickness $(d)$ of $38 \mu \mathrm{m}$ (thickness was obtained by a stylus profilometer) and an area (A) of $144 \mathrm{~mm}^{2}$. From the analysis we observed that the dielectric constant of the material decreases with increasing frequency, as shown in Figure 5c. At very low impedance, such as for at high frequency $(1 \mathrm{MHz})$, the dielectric constant of the material is 3.5 , and for high impedance $(100 \mathrm{~Hz})$ the value of the dielectric constant is 7.7. The high and low value of the dielectric constant in the different frequencies could be due to the ionic and electronic polarization occurring in the material. This relatively low permittivity suggests that $\beta$-Gly/CS is a good candidate for use in high-performance sensors. The piezoelectric voltage coefficient $\left(g\right.$ in $\left.\mathrm{Vm} \mathrm{N}^{-1}\right)$, defined by induced voltage under applied stress, is crucial to obtain a sensor with the desired performance: $g_{i j}=d_{i j} / \varepsilon_{r}$, where $g$ is inversely proportional to the dielectric constant. Materials such as PZT have a higher piezoelectric strain coefficient $\left(d_{i j}\right)$; however, they also have higher dielectric constants. ${ }^{38}$ In organic materials, due to lower values of the piezoelectric coefficient, a low dielectric constant is an advantage as it led to higher voltage outputs.

The energy loss in the piezoelectric generation of the material was measured using the loss tangent $(\tan \delta)$ using the following expression

$$
\text { loss factor } \tan \delta=\frac{G}{\omega C}
$$

Here, $G$ is the conductance of the material (shown in Figure S2 in the Supporting Information), $\omega$ is the angular frequency, and $C$ is the corresponding capacitance (shown in Figure 5a). The results show that the loss factor varies between 0.18 and 0.08 in the frequency range from $100 \mathrm{~Hz}$ to $1 \mathrm{MHz}$. At very low impedance, such as for at high frequency $(1 \mathrm{MHz})$, the loss factor of the material is 0.08 . The observed value of the 

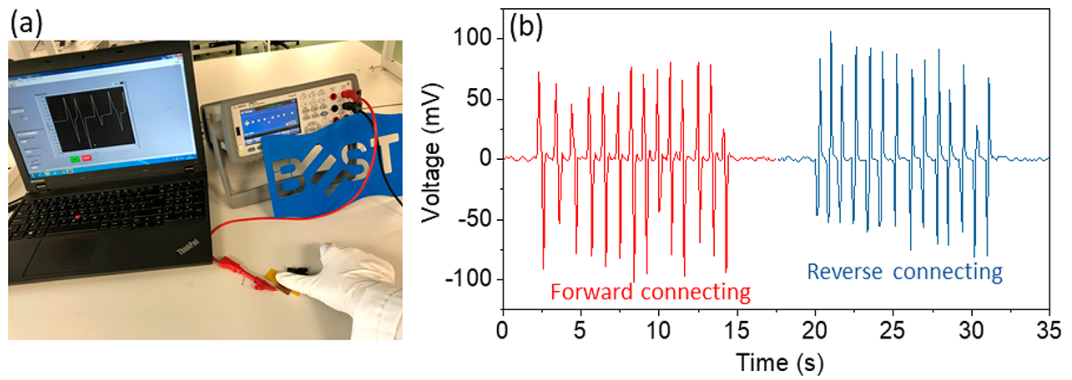

Figure 6. (a) Experimental arrangement for evaluation of the $\beta$-Gly/CS-based pressure sensor. (b) Sensor output when it was touched by hand under forward and reverse connections.

(a)
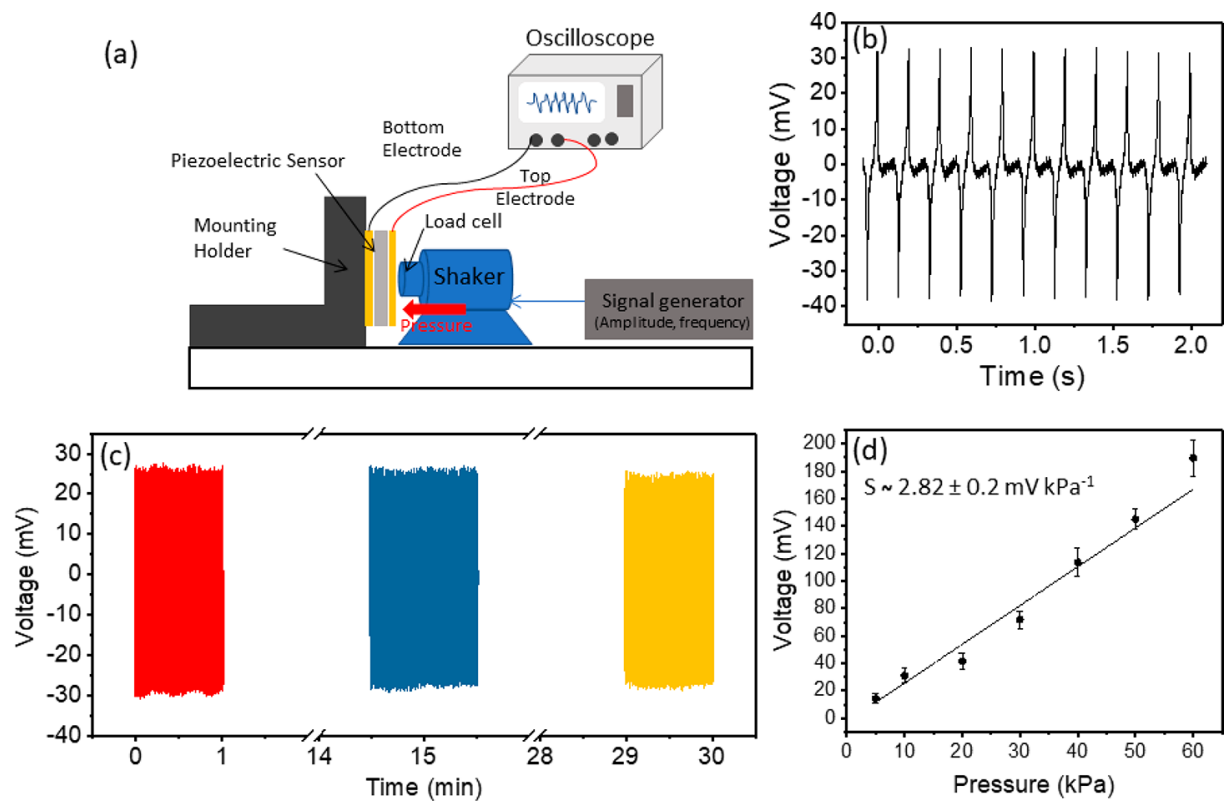

Figure 7. (a) Schematic of the characterization setup consisting of a shaker/vibration generator for measuring the sensor's sensitivity. (b) Output voltages of the $\beta$-Gly/CS sensor under a $10 \mathrm{kPa}$ applied pressure. (c) Cyclic measurement of the output voltage of the sensor for 9000 times repeated measurement at a pressure of $10 \mathrm{kPa}$ and $5 \mathrm{~Hz}$ frequency. (d) Piezoelectric sensitivity of the sensor as a function of applied pressure.

loss factor is comparable with the loss factor of the piezoelectric $\gamma$-phase of the glycine crystal. ${ }^{39}$ The observed ionic conductivity, dielectric properties, capacitance, and loss factor of the device show that the fabricated device could have excellent applicability in pressure-sensing applications, as we discuss in the following section.

3.3. Piezoelectric Characteristics of the $\beta$-Gly/CS Sensor. The piezoelectric output voltage performance and sensitivity of the $\beta$-Gly/CS-based sensor were measured to determine their suitability in pressure-sensing applications. Figure $6 \mathrm{a}$ and $6 \mathrm{~b}$ shows the experimental setup and the output voltage generated from the device when it was touched by hand. A repeat compression and release process showed the sensor responding uniformly with periodic positive and negative alterations (Figure 6b). In addition, the output voltage was measured after reversing the electrode connections (Figure $6 \mathrm{~b}$ ) to demonstrate that the output voltage is truly from the piezoelectric effect in the $\beta$-Gly/CS film.

The charge generated by the $\beta$-Gly/CS device was measured as a function of the dynamic pressure using a TIRA shaker (Figure $7 \mathrm{a}$ ). Figure $7 \mathrm{~b}$ shows the output voltage generated from the device under a dynamic pressure of $10 \mathrm{kPa}$ and $5 \mathrm{~Hz}$. The voltage generation is quite stable and repeatable with fast time response $(<100 \mathrm{~ms})$. The cycling stability of the $\beta$-Gly/
CS sensor device is confirmed under a constant pressure for more than 9000 cycles (Figure 7c). The produced voltage is measured with a 15 min interval and shows a good stability of signal over time. The sensitivity of the fabricated pressure sensor is investigated for the pressure range from 5 to $60 \mathrm{kPa}$. We observed that the generated voltage linearly increases with the applied compressive pressure as shown in Figure $7 \mathrm{~d}$. For each applied pressure, the output was measured from five devices, and the average response and standard deviation are calculated (Figure $7 \mathrm{~d}$ ). The measured sensitivity of the $\beta$-Gly/ CS sensor is $2.82 \pm 0.2 \mathrm{mV} \mathrm{kPa}^{-1}$, which was evaluated from the slope of the curve in Figure $7 \mathrm{~d}$. A comparison of the piezoelectric sensitivity of the $\beta$-Gly/CS device with other reported nondegradable piezoelectric polymers has been presented in Table S1.

The compression of a piezoelectric film by an external pressure brings charge separation in the composite film and generates a noticeable voltage between two electrodes. The overall piezoelectric response is the combined result of different polarization directions as each spherulite shows radial crystallization. In glycine, each molecule has a local dipole moment directed from the amino- to the carboxy-terminal direction and spontaneous polarization of glycine crystals comes from summation of the permanent dipole moments of 
glycine molecules in the volume. ${ }^{40}$ When an external pressure is applied to the device, each crystal undergoes deformation and strong net polarization is created due to movement of dipoles inside the crystalline structure from their equilibrium positions. This movement generates a potential difference in the film and between the top and the bottom electrodes and shows up as the piezoelectric voltage. When the mechanical pressure is released, the free charges flow back to the electrodes and produce a signal in the opposite direction. Thus, applying a higher compressive pressure leads to larger deformation of crystals and induces an enhanced piezopotential in the $\beta$-Gly/CS film.

It is interesting to note that the piezoelectric output opencircuit voltage (Figure 8) varies with the applied frequencies,

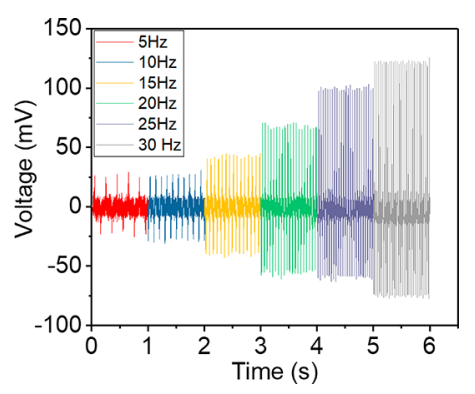

Figure 8. Piezoelectric output voltages of a $\beta$-Gly/CS sensor under a $10 \mathrm{kPa}$ applied pressure at different frequencies $(5-30 \mathrm{~Hz})$.

indicating the dependence of the output responses on the applied strain rate. At lower frequencies (i.e., $5 \mathrm{~Hz}$ ) the peak to peak voltage output from the $\beta$-Gly/CS is small (about 70 $\mathrm{mV}$ ). However, as the vibration frequency increases to $30 \mathrm{~Hz}$, the peak to peak voltage output increases to more than 200 $\mathrm{mV}$. This behavior can be due to the low stiffness of $\beta$-glycine crystals relative to ceramic piezoelectrics, and thus, the dynamic modulus increases with the frequency. The elastic constants for glycine crystals (Young's modulus $15 \mathrm{GPa}^{17}$ ) and chitosan film (Young's modulus $1.5 \mathrm{GPa}^{41}$ ) are smaller than typical inorganic piezoelectric materials such as PZT (Young's modulus $63 \mathrm{GPa}){ }^{42}$ Therefore, the elastic modulus of the $\beta$ Gly/CS composite film should be a value between 1.5 and 15 GPa. Because the piezoelectric voltage is known to be proportional to the elastic modulus of piezoelectric materials, an increase of the frequency of the applied vibration results in an increase of the signal generated by $\beta$-Gly/CS. This frequency-dependent increase in the piezoelectric output could find application in accelerometers and vibrometers. ${ }^{43}$

3.4. Biodegradability of the $\beta$-Gly/CS Sensor. In this work, nondegradable electrodes were used to characterize the piezoelectricity of $\beta$-Gly/CS due to the standard fabrication process and durability. Due to the biodegradable nature of the materials (glycine and chitosan) used in this work, it is important to evaluate the degradation rate of the sensor. In this regard, $\mathrm{Mg}$ as a biodegradable electrode ${ }^{1}$ was deposited on the $\beta$-Gly/CS film to fabricate a fully biodegradable sensor device. The solubility of the biodegradable sensor was evaluated in phosphate-buffered saline (PBS) solution ( $\mathrm{pH} 7.4)$. Figure S3 shows the biodegradability of the device with $\mathrm{Mg}$ electrodes in PBS solution at room temperature. Mg electrodes degradation occurs within the first minutes of immersing the device in the PBS medium, and Gly/Cs film dissolved in the solution a few days after (Figure S3). Thus, the fabricated device could be used as a single-use disposable sensor for ex-vivo applications such as monitoring pressure under the compression bandages. Nonetheless, with suitable encapsulation the sensors could be used for longer periods in applications such as wearable systems. For example, the whole sensor structure could be encapsulated with a thin layer $(100 \mu \mathrm{m})$ of biodegradable polymers with tunable degradation rates such as poly(L-lactideco-glycolide) (PLGA), which is resistant to water for up to 30 days. ${ }^{44}$

The piezoelectric performance of the sensor can be further improved to suit several practical applications. As mentioned above, glycine crystals have a permanent polarization due to the permanent dipole moment of glycine molecules and does not require a high electrical voltage (poling) to induce piezoelectric properties like electroactive polymers. It has been reported recently that $\beta$-glycine crystals have a high shear piezoelectric coefficient in the range of $178 \mathrm{pm} \mathrm{V}^{-1},{ }^{17}$ which is much higher than most of the biological piezoelectric materials and is similar to piezoceramics. However, piezoelectricity is a third-rank tensor property and is known to be dependent on crystallographic directions. This means that the orientation of the crystals in the film has a significant effect on the overall piezoelectric response of the sensor. Therefore, the piezoelectric response of the $\beta$-Gly/CS film is expected to increase remarkably by controlling the crystallization process or upon film poling. Further fabrication process development such as growing crystal under an external electric field could increase the film output voltage sensitivity by growing crystals in a unidirectional crystallography orientation in the entire film. ${ }^{45}$ Electrodes could also be applied with more simple techniques such as printing and using liquid metals that are highly conformable for wearable applications. ${ }^{46}$ In addition, due to the ferroelectric nature of glycine $\beta$-phase, ${ }^{22}$ poling could result in a uniform polarization of the entire film, which is not possible in most of the other piezoelectric biomaterials such as peptide nanotubes and cellulose fibrils. Fabrication of a multilayer sensor is another way to increase the sensitivity of the device. ${ }^{47}$ The flexible biocompatible pressure sensor presented here with good sensitivity under low-range pressure can be useful for applications such as continuous monitoring of sub-bandage pressure and tissue-swelling pressure.

\section{CONCLUSION}

The simple strategy for fabrication of a biocompatible and flexible piezoelectric pressure sensor made of biodegradable glycine and chitosan film presented in this paper could be useful for monitoring force/pressure in bioapplications. The chitosan enhances the flexibility of the brittle glycine crystals and could control the polymorph selectivity of the glycine molecules as well. The $\beta$-Gly/CS sensor relies on piezoelectricity, which allows the device to generate electrical output upon applied pressure. The sensor could produce a $190 \mathrm{mV}$ output voltage under $60 \mathrm{kPa}$ pressure with a sensitivity of 2.82 $\pm 0.2 \mathrm{mV} \mathrm{kPa}^{-1}$. The pressure sensor has a stable signal after 9000 cycles. The obtained results suggest that the glycinechitosan composite is a promising new piezoelectric material for sensors application. Further, the room-temperature and simple water-based solution fabrication process and biodegradability make the sensor an attractive alternative for eco-friendly sensor applications, particularly in health applications where it is critical to maintain hygiene conditions. The fabrication process can be studied further to control and optimize the crystals structure and enhance the piezoelectric properties of 
the films. Furthermore, the piezoelectricity of $\beta$-Gly/CS could be employed to generate voltage from biological deformations to produce useful electrical stimulation for tissue repair/ regeneration such as electrical stimulation of wound healing. As demonstrated in the literature, ${ }^{48}$ the external electric field (even as low as $12.5 \mathrm{mV} / \mathrm{mm}$ ) can effect cell migration toward the wound area and accelerate the healing process.

\section{ASSOCIATED CONTENT}

\section{SI Supporting Information}

The Supporting Information is available free of charge at https://pubs.acs.org/doi/10.1021/acsami.9b21052.

XRD pattern of the glycine-chitosan film obtained from various glycine contents in the chitosan solution; variation of the conductance of the $\beta$-Gly/CS film with frequency; optical images showing the biodegradability of the piezoelectric $\beta$-Gly/CS pressure sensor in the PBS solution with time; comparison of the piezoelectric sensitivity of $\beta$-Gly/CS sensor with reported nondegradable flexible piezoelectric polymers (PDF)

Voltage generated from the glycine/chitosan film during hand pressing (MP4)

\section{AUTHOR INFORMATION}

\section{Corresponding Author}

Ravinder Dahiya - Bendable Electronics and Sensing Technologies Group, James Watt School of Engineering, University of Glasgow, Glasgow G12 8QQ, United Kingdom; (1) orcid.org/0000-0002-3858-3841; Phone: +44 (0) 141 330 5653; Email: Ravinder.Dahiya@glasgow.ac.uk

\section{Authors}

Ensieh S. Hosseini - Bendable Electronics and Sensing Technologies Group, James Watt School of Engineering, University of Glasgow, Glasgow G12 8QQ United Kingdom

Libu Manjakkal - Bendable Electronics and Sensing Technologies Group, James Watt School of Engineering, University of Glasgow, Glasgow G12 8QQ United Kingdom

Dhayalan Shakthivel - Bendable Electronics and Sensing Technologies Group, James Watt School of Engineering, University of Glasgow, Glasgow G12 8QQ, United Kingdom

Complete contact information is available at:

https://pubs.acs.org/10.1021/acsami.9b21052

\section{Author Contributions}

E.S.H. and R.D. planned this work; E.S.H. fabricated the piezoelectric pressure sensor and performed analysis. L.M. and D.S. contributed to the impedance spectroscopic analysis and structural characterization of the device. E.S.H. and R.D. analyzed the data and wrote the manuscript. R.D. provided the overall supervision of this work.

\section{Notes}

The authors declare no competing financial interest.

\section{ACKNOWLEDGMENTS}

This work was supported by the European Commission through ELECTRO-HEAL (H2020-MSCA-IF-2016-753633) and the Engineering and Physical Sciences Research Council (EPSRC) through an Engineering Fellowship for Growth (EP/ R029644/1 and EP/M002527/1). The authors are thankful for the support received for this work from the James Watt Nanofabrication Centre (JWNC).

\section{REFERENCES}

(1) Boutry, C. M.; Nguyen, A.; Lawal, Q. O.; Chortos, A.; RondeauGagné, S.; Bao, Z. A Sensitive and BiodegradablePressure Sensor Array for Cardiovascular Monitoring. Adv. Mater. 2015, 27, 69546961.

(2) Garcia Nunez, C.; Manjakkal, L.; Dahiya, R. Energy Autonomous Electronic Skin. npj Flex. Electron. 2019, 3, 1-24.

(3) Yogeswaran, N.; Navaraj, W.; Gupta, S.; Liu, F.; Vinciguerra, V.; Lorenzelli, L.; Dahiya, R. Piezoelectric Graphene Field Effect Transistor Pressure Sensors for Tactile Sensing. Appl. Phys. Lett. 2018, 113, 014102.

(4) Saito, Y.; Takao, H.; Tani, T.; Nonoyama, T.; Takatori, K.; Homma, T.; Nagaya, T.; Nakamura, M. Lead-free Piezoceramics. Nature 2004, 432, 84-87.

(5) Mehmood, N.; Hariz, A.; Templeton, S.; Voelcker, N. H. An Improved Flexible Telemetry System to Autonomously Monitor Subbandage Pressure and Wound Moisture. Sensors 2014, 14, 2177021790.

(6) Hosseini, E. S.; Manjakkal, L.; Dahiya, R. Bio-Organic Glycine Based Flexible Piezoelectric Stress Sensor for Wound Monitoring. 2018 IEEE Sensors, New Delhi, IEEE, 2018; pp 1-4.

(7) Nguyen, V.; Zhu, R.; Jenkins, K.; Yang, R. Self-assembly of Diphenylalanine Peptide with Controlled Polarization for Power Generation. Nat. Commun. 2016, 7, 13566.

(8) Minary-Jolandan, M.; Yu, M.-F. Nanomechanical Heterogeneity in the Gap and Overlap Regions of Type I Collagen Fibrils with Implications for Bone Heterogeneity. Biomacromolecules 2009, 10, $2565-2570$.

(9) Heredia, A.; Meunier, V.; Bdikin, I. K.; Gracio, J.; Balke, N.; Jesse, S.; Tselev, A.; Agarwal, P. K.; Sumpter, B. G.; Kalinin, S. V. Nanoscale Ferroelectricity in Crystalline $\gamma$-glycine. Adv. Funct. Mater. 2012, 22, 2996-3003.

(10) Rajala, S.; Siponkoski, T.; Sarlin, E.; Mettänen, M.; Vuoriluoto, M.; Pammo, A.; Juuti, J.; Rojas, O. J.; Franssila, S.; Tuukkanen, S. Cellulose Nanofibril Film as a Piezoelectric Sensor Material. ACS Appl. Mater. Interfaces 2016, 8, 15607-15614.

(11) Lee, B. Y.; Zhang, J.; Zueger, C.; Chung, W.-J.; Yoo, S. Y.; Wang, E.; Meyer, J.; Ramesh, R.; Lee, S.-W. Virus-based Piezoelectric Energy Generation. Nat. Nanotechnol. 2012, 7, 351-356.

(12) Lee, J.-H.; Heo, K.; Schulz-Schönhagen, K.; Lee, J. H.; Desai, M. S.; Jin, H.-E.; Lee, S.-W. Diphenylalanine Peptide Nanotube Energy Harvesters. ACS Nano 2018, 12, 8138-8144.

(13) Vale, N.; Ferreira, A.; Matos, J.; Fresco, P.; Gouveia, M. Amino Acids in the Development of Prodrugs. Molecules 2018, 23, 2318.

(14) Perlovich, G.; Hansen, L. K.; Bauer-Brandl, A. The Polymorphism of Glycine. Thermochemical and Structural Aspects. J. Therm. Anal. Calorim. 2001, 66, 699-715.

(15) Dawson, A.; Allan, D. R.; Belmonte, S. A.; Clark, S. J.; David, W. I.; McGregor, P. A.; Parsons, S.; Pulham, C. R.; Sawyer, L. Effect of High Pressure on the Crystal Structures of Polymorphs of Glycine. Cryst. Growth Des. 2005, 5, 1415-1427.

(16) Seyedhosseini, E.; Bdikin, I.; Ivanov, M.; Vasileva, D.; Kudryavtsev, A.; Rodriguez, B.; Kholkin, A. Tip-induced Domain Structures and Polarization Switching in Ferroelectric Amino Acid Glycine. J. Appl. Phys. 2015, 118, 072008.

(17) Guerin, S.; Stapleton, A.; Chovan, D.; Mouras, R.; Gleeson, M.; McKeown, C.; Noor, M. R.; Silien, C.; Rhen, F. M.; Kholkin, A. L. Control of Piezoelectricity in Amino acids by Supramolecular Packing. Nat. Mater. 2018, 17, 180-186.

(18) Hamilton, B. D.; Hillmyer, M. A.; Ward, M. D. Glycine Polymorphism in Nanoscale Crystallization Chambers. Cryst. Growth Des. 2008, 8, 3368-3375.

(19) Jiang, Q.; Ward, M. D. Crystallization Under Nanoscale Confinement. Chem. Soc. Rev. 2014, 43, 2066-2079. 
(20) Lee, I. S.; Kim, K. T.; Lee, A. Y.; Myerson, A. S. Concomitant Crystallization of Glycine on Patterned Substrates: The Effect of $\mathrm{pH}$ on the Polymorphic Outcome. Cryst. Growth Des. 2008, 8, 108-113.

(21) Seyedhosseini, E.; Ivanov, M.; Bystrov, V.; Bdikin, I.; Zelenovskiy, P.; Shur, V. Y.; Kudryavtsev, A.; Mishina, E. D.; Sigov, A. S.; Kholkin, A. L. Growth and Nonlinear Optical Properties of $\beta$ glycine Crystals Grown on Pt Substrates. Cryst. Growth Des. 2014, 14, 2831-2837.

(22) Seyedhosseini, E.; Romanyuk, K.; Vasileva, D.; Vasilev, S.; Nuraeva, A.; Zelenovskiy, P.; Ivanov, M.; Morozovska, A. N.; Shur, V. Y.; Lu, H. Self-assembly of Organic Ferroelectrics by Evaporative Dewetting: A Case of $\beta$-Glycine. ACS Appl. Mater. Interfaces 2017, 9, 20029-20037.

(23) Kafi, M. A.; Aktar, K.; Todo, M.; Dahiya, R. Engineered Chitosan for Improved 3D Tissue Growth through Paxillin-FAK-ERK Activation. Regen. Biomater. 2019, 1-11.

(24) Kafi, M. A.; Paul, A.; Vilouras, A.; Dahiya, R. Mesoporous Chitosan based Conformable and Resorbable Biostrip for Dopamine Detection. Biosens. Bioelectron. 2020, 147, 111781.

(25) Rodríguez-Vázquez, M.; Vega-Ruiz, B.; Ramos-Zúñiga, R.; Saldaña-Koppel, D. A.; Quiñones-Olvera, L. F. Chitosan and its Potential Use as a Scaffold for Tissue Engineering in Regenerative Medicine. BioMed Res. Int. 2015, 2015, 821279.

(26) Rinaudo, M. Chitin and Chitosan: Properties and Applications. Prog. Polym. Sci. 2006, 31, 603-632.

(27) Ueno, H.; Mori, T.; Fujinaga, T. Topical Formulations and Wound Healing Applications of Chitosan. Adv. Drug Delivery Rev. 2001, 52, 105-115.

(28) Kafi, M. A.; Paul, A.; Vilouras, A.; Hosseini, E. S.; Dahiya, R. S. Chitosan-Graphene Oxide based Ultra-thin and Flexible Sensor for Diabetic Wound Monitoring. IEEE Sens. J. 2019, 1-8.

(29) Silva, C. C.; Lima, C. G. A.; Pinheiro, A. G.; Goes, J. C.; Figueiro, S. D.; Sombra, A. S. B. On the Piezoelectricity of Collagenchitosan Films. Phys. Chem. Chem. Phys. 2001, 3, 4154-4157.

(30) Mucha, M.; Piekielna, J.; Wieczorek, A. Characterisation and Morphology of Biodegradable Chitosan/Synthetic Polymer Blends. Macromolecular symposia; Wiley Online Library, 1999; pp 391-412.

(31) Kumar, R.; Prakash, K.; Cheang, P.; Gower, L.; Khor, K. Chitosan-mediated Crystallization and Assembly of Hydroxyapatite Nanoparticles into Hybrid Nanostructured Films. J. R. Soc., Interface 2008, 5, 427-439.

(32) Crist, B.; Schultz, J. M. Polymer Spherulites: A Critical Review. Prog. Polym. Sci. 2016, 56, 1-63.

(33) Dong, Y.; Sakurai, K.; Wu, Y.; Kondo, Y. Spherulite Morphology Studies of $\mathrm{N}$-alkyl Chitosan Films Cast from Formic Acid Solutions. J. Polym. Sci., Part B: Polym. Phys. 2003, 41, 20332038.

(34) Ibrahim, M. A.; Gawad, A. E.-D. A. Spectroscopic Analyses of Chitosan Interactions with Amino Acids. J. Comput. Theor. Nanosci. 2012, 9, 1120-1124.

(35) Gránásy, L.; Pusztai, T.; Tegze, G.; Warren, J. A.; Douglas, J. F. Growth and Form of Spherulites. Phys. Rev. E 2005, 72, 011605.

(36) Ulčinas, A.; Butler, M.; Heppenstall-Butler, M.; Singleton, S.; Miles, M. Direct Observation of Spherulitic Growth Stages of $\mathrm{CaCO} 3$ in a Poly (acrylic acid)-chitosan System: in Situ SPM Study. J. Cryst. Growth 2007, 307, 378-385.

(37) Tylczyński, Z.; Busz, P. Low-temperature Phase Transition in $\gamma$ glycine Single Crystal. Pyroelectric, Piezoelectric, Dielectric and Elastic Properties. Mater. Chem. Phys. 2016, 183, 254-262.

(38) Tressler, J. F.; Alkoy, S.; Newnham, R. E. Piezoelectric Sensors and Sensor Materials. J. Electroceram. 1998, 2, 257-272.

(39) Kumar, R. A.; Vizhi, R. E.; Vijayan, N.; Babu, D. R. Structural, Dielectric and Piezoelectric Properties of Nonlinear Optical $\gamma$-glycine Single Crystals. Phys. B 2011, 406, 2594-2600.

(40) Bystrov, V.; Seyedhosseini, E.; Kopyl, S.; Bdikin, I.; Kholkin, A. Piezoelectricity and Ferroelectricity in Biomaterials: Molecular Modeling and Piezoresponse Force Microscopy Measurements. J. Appl. Phys. 2014, 116, 066803.
(41) Aryaei, A.; Jayatissa, A. H.; Jayasuriya, A. C. Nano and Micro Mechanical Properties of Uncross-linked and Cross-linked Chitosan Films. J. Mech. Behav. Biomed. 2012, 5, 82-89.

(42) Low, T.; Guo, W. Modeling of a Three-layer Piezoelectric Bimorph Beam with Hysteresis. J. Microelectromech. Syst. 1995, 4, 230-237.

(43) Persano, L.; Dagdeviren, C.; Su, Y.; Zhang, Y.; Girardo, S.; Pisignano, D.; Huang, Y.; Rogers, J. A. High Performance Piezoelectric Devices based on Aligned Arrays of Nanofibers of Poly (vinylidenefluoride-co-trifluoroethylene). Nat. Commun. 2013, 4, 1633.

(44) Zheng, Q.; Zou, Y.; Zhang, Y.; Liu, Z.; Shi, B.; Wang, X.; Jin, Y.; Ouyang, H.; Li, Z.; Wang, Z. L. Biodegradable Triboelectric Nanogenerator as a Life-time Designed Implantable Power Source. Sci. Adv. 2016, 2, e1501478.

(45) Nanev, C. N.; Penkova, A. Nucleation of Lysozyme Crystals Under External Electric and Ultrasonic Fields. J. Cryst. Growth 2001, 232, 285-293.

(46) Yang, J.; Cheng, W.; Kalantar-Zadeh, K. Electronic Skins Based on Liquid Metals. Proc. IEEE 2019, 107, 2168-2184.

(47) Curry, E. J.; Ke, K.; Chorsi, M. T.; Wrobel, K. S.; Miller, A. N.; Patel, A.; Kim, I.; Feng, J.; Yue, L.; Wu, Q. Biodegradable Piezoelectric Force Sensor. Proc. Natl. Acad. Sci. U. S. A. 2018, 115, 909-914.

(48) Zhao, M.; Song, B.; Pu, J.; Wada, T.; Reid, B.; Tai, G.; Wang, F.; Guo, A.; Walczysko, P.; Gu, Y.; Sasaki, T. Electrical Signals Control Wound Healing Through Phosphatidylinositol-3-OH kinase$\gamma$ and PTEN. Nature 2006, 442, 457-460. 